\title{
Fracionamento do fósforo avaliação de extratores do P-disponível em solos da llha de Fernando de Noronha ${ }^{1}$
}

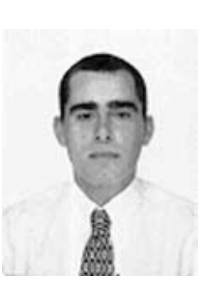

\author{
Alexandre T. da Rocha ${ }^{2}$, Gustavo P. Duda ${ }^{3}$, Clístenes W. A. Nascimento ${ }^{4}$ \& Mateus R. Ribeiro ${ }^{4}$
}

\footnotetext{
${ }^{1}$ Parte da Dissertação de Mestrado em Ciência do Solo apresentada na Universidade Federal Rural de Pernambuco

${ }^{2}$ Rua Alm. José Dias Fernandes 102/101, Piedade, Jaboatão dos Guararapes, PE. CEP: 54400-300, Fone (81) 99692103 , E-mail: altarocha@universia.com.br (Foto)

${ }^{3}$ DCA/ESAM. CEP: 59625-900, Mossoró, RN. Fone: (84) 315 0647, E-mail: gpduda@esam.br

${ }^{4}$ DEPA/UFRPE. CEP: 52171-900, Recife, PE. Fone (81) 3302 1220, E-mail: cwan@bol.com.br
}

Protocolo 6 - 28/1/2004 - Aprovado em 18/10/2004

\begin{abstract}
Resumo: Os solos de Fernando de Noronha, PE, possuem alta fertilidade natural e elevados teores de fósforo (P) em decorrência do material de origem vulcânica e da deposição de guano. Com este trabalho objetivou-se, mediante o fracionamento de $\mathrm{P}$, avaliar a distribuição das frações orgânicas e inorgânicas em perfis de solo, auxiliando nos estudos pedogenéticos e na identificação das formas disponíveis do nutriente nesses solos; para isto, utilizam-se 24 amostras de solo provenientes de cinco perfis coletados em uma área agrícola com extensão de aproximadamente 16 ha, as quais foram submetidas aos fracionamentos de $P$ orgânico e inorgânico. As frações obtidas foram correlacionadas entre si e com características químicas dos solos. Os resultados demonstram que os altos teores de fósforo observados nos solos de Fernando de Noronha são decorrentes, sobretudo, do material de origem, ressaltando-se que o fósforo orgânico foi menos importante que as formas inorgânicas na contribuição para o fósforo total desses solos. P-Fe e P-Ca são as frações predominantes nos perfis estudados. Os extratores Mehlich 3 e Olsen, melhor correlacionados com as frações mais lábeis de $P$, foram mais eficientes que o Mehlich 1 para estimar o P disponível nos solos estudados.
\end{abstract}

Palavras-chave: frações de P, fósforo orgânico, material de origem

\section{Phosphorus fractionation and evaluation of available-P extractors in soils of Fernando de Noronha Island}

\begin{abstract}
The soils of Fernando de Noronha Island present high natural soil fertility and high contents of phosphorus (P) derived from vulcanic soil parent material and probably "guano" deposits. Soil phosphorus fractionation was carried out in these soils in order to define the distribution of organic and inorganic fractions in soil horizon, providing information for studies on soil genesis and prediction of plant available $P$ forms in soils. Five soil profiles were selected and twenty four soil samples were analysed aiming to determine organic and inorganic $P$ fractions, which were correlated with each other as well as with soil chemical properties. The results showed that the high $\mathrm{P}$ contents found in the soil samples were derived mainly from the soil parent material and, as a consequence, the organic phosphorus was less important than inorganic forms in contributing to the total phosphorus in such soils. P-Ca and P-Fe fractions are the most important chemical forms in the soil samples. The Mehlich-3 ond Olsen extractors, which were better correlated to labile fractions of phosphorus, were more efficient than Mehlich-1 for predicting the available phosphorus in the soils.
\end{abstract}

Key words: P fractions, orgânic phosphorus, parent material

\section{INTRODUÇÃO}

A disponibilidade de fósforo $(\mathrm{P})$ nos solos é regulada por processos geoquímicos e biológicos. Em ecossistemas naturais, os processos geoquímicos podem determinar, a longo prazo, a disponibilidade de $\mathrm{P}$ nos solos mas, a curto prazo, os processos biológicos merecem destaque em virtude da maior utilização deste elemento pelas plantas, via mineralização da matéria orgânica do solo (Cross \& Schlesinger, 1995; Chen et al., 2003). No primeiro caso, as formas de $\mathrm{P}$ e a variabilidade dos 
teores de $\mathrm{P}$, ao longo do perfil, são bastante influenciadas pelos processos pedogenéticos (Walker \& Syers, 1976; Smeck, 1985; Cassagne et al., 2000).

Visto que o conteúdo total de $\mathrm{P}$ no solo não reflete sua disponibilidade para as plantas, torna-se imprescindível avaliar o P disponível para as plantas. Para isto, diversos extratores têm sido utilizados como, por exemplo, água, Bray 1, Olsen, Mehlich 1, Mehlich 2, Mehlich 3, $\mathrm{NH}_{4} \mathrm{HCO}_{3}+\mathrm{DTPA}$, resina aniônica e diluição isotópica (Tan, 1996; Novais \& Smith, 1999).

Tradicionalmente, os métodos de avaliação da fertilidade do solo enfocam a fração inorgânica de P como indicadora da disponibilidade deste nutriente para as culturas; contudo, sabese que a fração orgânica contribui significativamente para o fornecimento de $\mathrm{P}$ às plantas, pelo processo de mineralização (Stevenson, 1994; Canellas et al., 1999; Rheinheimer et al., 1999). Duda (2000) trabalhando com diversas amostras de solos do Brasil, observou que 7 a $83 \%$ do P total do solo se encontram na forma orgânica e 5 a $86 \%$ deste podem estar na fração orgânica lábil (Guerra, 1996).

De acordo com Silva \& van Raij (1999) para entender melhor a dinâmica do fósforo em solos, é necessário conhecer as diferentes frações do elemento, mediante a utilização seqüencial de diferentes soluções extratoras. O conhecimento da natureza e da distribuição dessas frações pode fornecer informações importantes para a avaliação da disponibilidade do fósforo no solo (Tiessen et al., 1984).

Os solos do arquipélago Fernando de Noronha apresentam teores extremamente elevados de fósforo disponível (Nascimento, 2002), que podem ser originados de duas fontes principais, ou seja, da deposição de guano, intensa em ilhas oceânicas, e da própria origem vulcânica da ilha, que possibilitou material de origem com teores naturalmente altos de fósforo. Nesta condição particular, é conveniente identificar a fonte responsável pelo fornecimento do $\mathrm{P}$ disponível e as frações do elemento nesses solos.

Diante do exposto, o objetivo deste trabalho foi estudar a distribuição das frações orgânicas e inorgânicas de $\mathrm{P}$ ao longo de perfis de solos da ilha principal (Ilha de Fernando de Noronha) do Arquipélago de Fernando de Noronha, com vistas a auxiliar nos estudos pedogenéticos e na identificação das formas de fósforo nos referidos solos.

\section{MATERIAL E MÉTODOS}

Os perfis estudados se situam na Ilha principal do Distrito Estadual de Fernando de Noronha, PE, na área agrícola do Projeto Noronha Terra, localizada nos arredores do açude da Ema, entre a pista de pouso do aeroporto e o morro do Pico, limitada ao longo do seu perímetro norte pela rodovia Transnoronha.

O Arquipélago de Fernando de Noronha localiza-se nas coordenadas $3^{\circ} 54^{\prime} \mathrm{S}$ de latitude e $32^{\circ} 25^{\prime} \mathrm{W}$ de longitude a 545 $\mathrm{km}$ de Recife, capital do Estado de Pernambuco, e a $360 \mathrm{~km}$ de Natal, capital do Estado do Rio Grande do Norte; formado pelo topo das montanhas de uma cordilheira de origem vulcânica, sua base se localiza a cerca de $4.000 \mathrm{~m}$ de profundidade, a Dorsa Mediana do Atlântico, e é constituído por 21 ilhas, rochedos e ilhotas, no total aproximado de $26 \mathrm{~km}^{2}$, tendo a ilha principal $17 \mathrm{~km}^{2}$ de área. O clima é tropical, com franco domínio oceânico e duas estações bem definidas, enquanto a pluviometria média é de $1.300 \mathrm{~mm}$ anuais, com período chuvoso entre março e julho e estiagem de agosto a fevereiro, com umidade relativa bastante constante, com média anual de $81 \%$ e temperatura média de $25,4{ }^{\circ} \mathrm{C}$ (Nascimento, 2002). A ilha se compõe de dois tipos de rocha, vulcânica e sedimentar, que se dividem nas formações Remédios, Quixaba e São José, em que os solos utilizados neste trabalho são desenvolvidos de tufos da formação Remédios. Segundo Nascimento (2002), esses solos têm alta fertilidade, com teores elevados de fósforo, cálcio, magnésio e baixíssimo teor de alumínio, com predomínio da textura argilosa.

Para as análises foram utilizadas 24 amostras de solo, correspondentes aos horizontes de 5 perfis (Tabela 1) coletadas em área agrícola com extensão de 16 ha. As descrições e coletas seguiram as normas e recomendações da Sociedade Brasileira de Ciência do Solo (Lemos \& Santos, 1996).

Tabela 1. Classes de solo coletadas em Fernando de Noronha

\begin{tabular}{|c|c|c|c|c|}
\hline Perfil $n^{\circ}$ & Classe de solo & Uso Atual & Horizontes & Prof. $(\mathrm{cm})$ \\
\hline \multirow[t]{5}{*}{1} & CAMBISSOLO & \multirow{5}{*}{$\begin{array}{l}\text { Milho e } \\
\text { hortaliças }\end{array}$} & Ap & $0-15$ \\
\hline & HÁPLICO & & $\mathrm{BA}$ & $15-40$ \\
\hline & Tb Eutrófico & & $\mathrm{Bi}_{1}$ & $40-73$ \\
\hline & vértico gleico & & $\mathrm{Bi}_{2}$ & $73-110$ \\
\hline & solódico & & $2 \mathrm{CVgn}$ & $110-134$ \\
\hline \multirow[t]{5}{*}{2} & CAMBISSOLO & \multirow{5}{*}{$\begin{array}{l}\text { Pastagem } \\
\text { nativa }\end{array}$} & A & $0-11$ \\
\hline & HÁPLICO & & $\mathrm{Bi}$ & $11-43$ \\
\hline & Sódico vértico & & $2 \mathrm{Cnv}_{1}$ & $43-68$ \\
\hline & & & $2 \mathrm{Cnv}_{2}$ & $68-95$ \\
\hline & & & $2 \mathrm{Cr} / \mathrm{R}$ & $95-130$ \\
\hline \multirow[t]{4}{*}{3} & CAMBISSOLO & \multirow{4}{*}{$\begin{array}{l}\text { Capim } \\
\text { elefante }\end{array}$} & Ap & $0-14$ \\
\hline & HÁPLICO & & $\mathrm{Bi}_{1}$ & $14-38$ \\
\hline & Ta Eutrófico & & $\mathrm{Bi}_{2}$ & $38-60$ \\
\hline & típico & & $\mathrm{C}$ & $60-130$ \\
\hline \multirow[t]{5}{*}{4} & CAMBISSOLO & \multirow{5}{*}{$\begin{array}{l}\text { Abóbora, } \\
\text { quiabo e } \\
\text { feijão }\end{array}$} & Apn & $0-20$ \\
\hline & HÁPLICO & & Bin & $20-48$ \\
\hline & Sódico vértico & & Binv & $48-72$ \\
\hline & gleico & & $2 \mathrm{CnV}_{1}$ & $72-108$ \\
\hline & & & $2 \mathrm{CnV}_{2}$ & $108-158$ \\
\hline \multirow[t]{5}{*}{5} & VERTISSOLO & \multirow[t]{5}{*}{ Milho } & Apn & $0-14$ \\
\hline & CROMADO & & $\mathrm{ACn}$ & $14-32$ \\
\hline & Sódico gleico & & $\mathrm{Cnv}$ & $32-53$ \\
\hline & & & $\mathrm{Cnvg}_{1}$ & $53-95$ \\
\hline & & & $\mathrm{Cnvg}_{2}$ & $95-155$ \\
\hline
\end{tabular}

Os perfis foram classificados segundo o Sistema Brasileiro de Classificação de Solos (EMBRAPA, 1999). As amostras de solos foram caracterizadas quimicamente (Tabela 2) utilizandose os métodos propostos pela EMBRAPA (1997); depois, elas foram submetidas ao fracionamento de fósforo orgânico (Po), obtendo-se a fração lábil, segundo Bowman \& Cole (1978), e as frações moderadamente lábil e moderadamente resistente, de acordo com Bowman (1989) observando-se as modificações realizadas por Guerra (1993) e Duda (2000).

$\mathrm{O} \mathrm{P}$ orgânico nas formas lábil, moderadamente lábil e moderadamente resistente, ou seja, solúvel em bicarbonato de 
Tabela 2. Características químicas e teor de argila dos perfis de Fernando de Noronha

\begin{tabular}{|c|c|c|c|c|c|c|c|c|c|c|}
\hline \multirow[t]{2}{*}{ Perfil ${ }^{*}$} & \multirow[t]{2}{*}{ Horiz. } & \multirow{2}{*}{$\begin{array}{c}\mathrm{pH} \\
\left(\mathrm{H}_{2} \mathrm{O}\right)\end{array}$} & $\mathrm{Ca}^{2+}$ & $\mathrm{Mg}^{2+}$ & $\mathrm{K}^{+}$ & $\mathrm{Na}^{+}$ & \multirow[t]{2}{*}{$\mathrm{Al}^{3+}$} & $\mathrm{P}$ & C.O & Argila \\
\hline & & & & & $\operatorname{mol}_{\mathrm{c}} \mathrm{k}$ & & & $\mathrm{mg} \mathrm{kg}^{-1}$ & \multicolumn{2}{|c|}{$\mathrm{g} \mathrm{kg}^{-1}$} \\
\hline \multirow{6}{*}{1} & Ap & 5,2 & 5,9 & 2,6 & 0,43 & 0,26 & 0,4 & 349 & 12,2 & 570 \\
\hline & BA & 5,3 & 3,7 & 2,1 & 0,17 & 0,29 & 0,5 & 270 & 10,5 & 510 \\
\hline & Bil & 5,7 & 3,7 & 3,1 & 0,05 & 0,32 & 0,2 & 338 & 4,5 & 520 \\
\hline & $\mathrm{Bi} 2$ & 6,1 & 2,7 & 4,8 & 0,03 & 0,86 & 0,0 & 145 & 3,0 & 570 \\
\hline & 2Cvgn & 6,0 & 15,3 & 17,7 & 0,05 & 4,78 & 0,0 & 29 & 3,5 & 710 \\
\hline & A & 5,8 & 14,4 & 4,0 & 0,59 & 0,37 & 0,1 & 270 & 14,8 & 590 \\
\hline \multirow{4}{*}{2} & $\mathrm{Bi}$ & 6,3 & 8,3 & 5,1 & 0,12 & 0,79 & 0,0 & 259 & 4,2 & 650 \\
\hline & $2 \mathrm{Cnv} 1$ & 6,4 & 47,4 & 24,2 & 0,04 & 6,02 & 0,0 & 31 & 3,0 & 710 \\
\hline & $2 \mathrm{Cnv} 2$ & 6,3 & 18,6 & 12,3 & 0,04 & 12,16 & 0,0 & 1002 & 2,4 & 710 \\
\hline & $2 \mathrm{Cr} / \mathrm{R}$ & 8,2 & 41,9 & 23,9 & 0,03 & 16,18 & 0,0 & 1053 & 1,4 & 370 \\
\hline \multirow{4}{*}{3} & A & 6,1 & 24,2 & 4,3 & 0,21 & 0,43 & 0,0 & 338 & 14,1 & 530 \\
\hline & Bil & 6,3 & 5,4 & 1,1 & 0,16 & 0,43 & 0,0 & 497 & 2,7 & 592 \\
\hline & $\mathrm{Bi} 2$ & 6,3 & 13,0 & 3,2 & 0,13 & 1,39 & 0,0 & 418 & 5,0 & 590 \\
\hline & $\mathrm{C}$ & 5,8 & 44,8 & 11,5 & 0,11 & 3,87 & 0,0 & 611 & 5,0 & 350 \\
\hline \multirow{5}{*}{4} & Apn & 6,1 & 6,5 & 1,5 & 0,40 & 1,78 & 0,0 & 346 & 18,4 & 370 \\
\hline & Bin & 6,6 & 3,9 & 2,1 & 0,16 & 1,67 & 0,0 & 401 & 5,1 & 460 \\
\hline & Binv & 6,9 & 5,7 & 5,1 & 0,06 & 3,45 & 0,0 & 74 & 3,0 & 650 \\
\hline & $2 \mathrm{Cnv} 1$ & 7,0 & 7,8 & 7,2 & 0,06 & 5,12 & 0,0 & 54 & 2,8 & 700 \\
\hline & $2 \mathrm{Cnv} 2$ & 7,7 & 6,4 & 5,6 & 0,05 & 6,06 & 0,0 & 54 & 3,5 & 720 \\
\hline \multirow{5}{*}{5} & Apn & 6,9 & 7,5 & 4,1 & 0,28 & 3,45 & 0,0 & 243 & 19,1 & 480 \\
\hline & Acn & 7,9 & 5,7 & 5,2 & 0,08 & 6,48 & 0,0 & 188 & 5,7 & 640 \\
\hline & $\mathrm{Cnv}$ & 8,2 & 5,5 & 5,6 & 0,11 & 6,79 & 0,0 & 228 & 3,4 & 700 \\
\hline & Cnvgl & 8,8 & 6,3 & 5,5 & 0,34 & 7,32 & 0,0 & 314 & 3,2 & 710 \\
\hline & $\mathrm{Cnvg}_{2}$ & 8,9 & 6,1 & 5,4 & 0,59 & 6,37 & 0,0 & 2,20 & 4,1 & 640 \\
\hline
\end{tabular}

* 1- CAMBISSOLO HÁPLICO Tb Eutrófico vértico gleico Solódico; 2- CAMBISSOLO HÁPLICO Sódico vértico; 3- CAMBISSOLO HÁPLICO Ta Eutrófico típico; 4- CAMBISSOLO HÁPLICO Sódico vértico gleico; 5- VERTISSOLO CROMADO Sódico gleico

sódio, ácido sulfúrico e hidróxido de sódio, respectivamente, foi obtido pela diferença entre o P inorgânico e o P total encontrado em cada um dos extratos obtidos; com isto, uma parte do extrato $(10 \mathrm{~mL})$ foi clarificada com $0,5 \mathrm{~cm}^{3}$ carvão ativo previamente purificado, para a obtenção do P inorgânico e a outra submetida a digestão com ácido perclórico concentrado na presença de cloreto de magnésio, para a obtenção do fósforo total em cada extrator (Duda, 2000) (Figura 1).

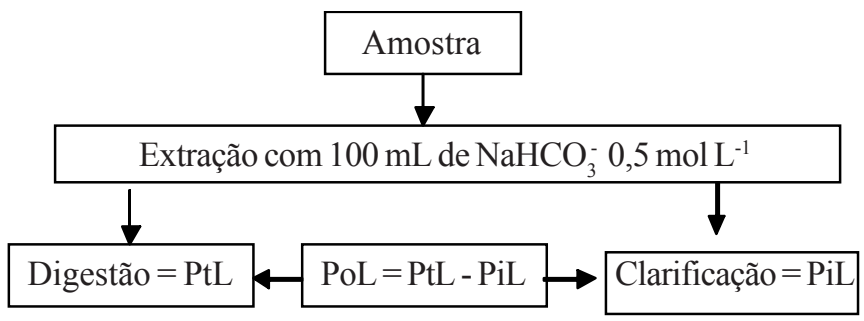

Figura 1. Esquema de extração e determinação de fósforo orgânico lábil conforme Bowman \& Cole (1978) em que PtLé fósforo total lábil, Pil é fósforo inorgânico lábil e Pol é fósforo orgânico lábil

Para evitar contaminação das amostras pelo $\mathrm{P}$ contido no carvão ativo, este último foi submetido a lavagens sucessivas, utilizando-se $\mathrm{HCl} 6 \mathrm{~mol} \mathrm{~L}^{-1}$, $\mathrm{NaOH} 0,5 \mathrm{~mol} \mathrm{~L}^{-1}$ e água destilada, obedecendo a relação 1:1:10 L, respectivamente, para cada 100 g de carvão ativo.
A fração Po lábil foi obtida utilizando-se $100 \mathrm{~mL}$ de $\mathrm{NaHCO}_{3}$ $0,5 \mathrm{~mol} \mathrm{~L}^{-1} \mathrm{com} \mathrm{pH}$ corrigido para 8,5 (imediatamente antes do uso) para cada $5 \mathrm{~g}$ de solo, de acordo com o esquema apresentado na figura abaixo.

A fração moderadamente lábil foi obtida após a adição de 3 $\mathrm{mL}$ de $\mathrm{H}_{2} \mathrm{SO}_{4} 18 \mathrm{~mol} \mathrm{~L}^{-1}$ e $47 \mathrm{~mL}$ de água à amostra de $2 \mathrm{~g}$ de solo. Após ligeira agitação, a solução sobrenadante foi filtrada e submetida à quantificação de $\mathrm{P}$ inorgânico e de $\mathrm{P}$ total solúvel em ácido e, para determinação da fração moderadamente resistente, adicionaram-se $100 \mathrm{~mL}$ de $\mathrm{NaOH} 0,5 \mathrm{~mol} \mathrm{~L}^{-1}$ ao solo remanescente no recipiente, deixando-o em banho maria a 80 ${ }^{\circ} \mathrm{C}$, durante $2 \mathrm{~h}$. A solução sobrenadante foi filtrada e submetida a quantificação de $\mathrm{P}$ inorgânico e de $\mathrm{P}$ total solúvel em álcali, enquanto o fósforo orgânico foi definido pelo somatório das frações orgânicas solúveis em ácido e em álcali.

Para o fracionamento do $\mathrm{P}$ inorgânico usou-se o procedimento proposto por Chang \& Jackson (1957), com algumas modificações sugeridas por Novais (1977) determinandose, assim, o P facilmente solúvel ("P- $\mathrm{H}_{2} \mathrm{O}$ "), $\mathrm{P}$ ligado a alumínio ( $\mathrm{P}-\mathrm{Al}), \mathrm{P}$ ligado a ferro (P-Fe) e o $\mathrm{P}$ ligado a cálcio (P-Ca), de acordo com o descrito abaixo:

\section{P facilmente solúvel:}

Colocou-se $0,5 \mathrm{~g}$ de solo para agitar em tubo de centrífuga contendo $25 \mathrm{~mL}$ de $\mathrm{NH}_{4} \mathrm{Cl} 1 \mathrm{~mol} \mathrm{~L}^{-1}$, durante $30 \mathrm{~min}$ a suspensão foi centrifugada a $1600 \mathrm{~g}$ e o sobrenadante coletado para a quantificação de fósforo. 


\section{P ligado a alumínio:}

À amostra no tubo foram adicionados $25 \mathrm{~mL}$ de $\mathrm{NH}_{4} \mathrm{~F} 0,5$ mol L-1, seguidos de agitação por $1 \mathrm{~h}$; após centrifugação, o extrato foi coletado para a dosagem de fósforo.

\section{$P$ ligado a ferro:}

A amostra de solo retida no tubo foi lavada duas vezes, com porções de $25 \mathrm{~mL}$ de uma solução saturada de $\mathrm{NaCl}$; em seguida, utilizaram-se $25 \mathrm{~mL}$ de $\mathrm{NaOH} 0,1 \mathrm{~mol} \mathrm{~L}^{-1}+\mathrm{NaCl} 1 \mathrm{~mol} \mathrm{~L}^{-1}$ sob agitação por $17 \mathrm{~h}$. A suspensão foi centrifugada a $1600 \mathrm{~g}$ durante 15 min e o procedimento repetido sucessivas vezes até obtenção de um extrato límpido, para posterior quantificação de P no extrato.

\section{P ligado a cálcio:}

A amostra de solo remanescente da extração anterior foi lavada duas vezes com porções de $25 \mathrm{~mL}$ de uma solução saturada de $\mathrm{NaCl}$. A extração foi efetuada utilizando-se $25 \mathrm{~mL}$ de $\mathrm{H}_{2} \mathrm{SO}_{4} 0,25 \mathrm{~mol} \mathrm{~L}^{-1}$ e $1 \mathrm{~h}$ de agitação; a suspensão foi centrifugada e o sobrenadante coletado para a quantificação de P; para esta fração foi feito o teste de não-interferência de íons de ferro tomando-se, para tal, duas alíquotas idênticas do extrato de $\mathrm{P}-\mathrm{Ca}$, em uma das quais foi adicionada uma solução padrão para promover uma concentração final de fósforo de $0,2 \mathrm{mg} \mathrm{L}^{-1}$, sendo o teor de fósforo determinado em ambas as amostras. A completa recuperação do P adicionado estabelece a não-interferência dos íons de ferro na solução; nos solos estudados não se detectou a interferência de íons de ferro nos extratos obtidos para determinação do P-cálcio.

Algumas amostras de solo de camadas superficiais apresentaram extratos tingidos pela matéria orgânica, fazendo-se necessário adicionar-lhes gotas de $\mathrm{H}_{2} \mathrm{SO}_{4} 1 \mathrm{~mol} \mathrm{~L}^{-1}$ até a floculação dos colóides orgânicos; em seguida, a solução foi centrifugada e o extrato, clarificado, teve o $\mathrm{pH}$ corrigido para 3 , visando posterior determinação.

Obteve-se o fósforo total do solo (Pt) segundo O'Halloran (1993). Os teores de fósforo disponíveis foram avaliados por três extratores químicos: Mehlich-1 (EMBRAPA,1997), Mehlich3 (Mehlich, 1984) e Olsen (Olsen et al., 1954). Todas as determinações de $\mathrm{P}$ deste trabalho foram realizadas por espectrofotometria, como descrito por Braga \& Defelipo (1974).

Os dados obtidos e os valores das características químicas das amostras de solo foram submetidos a análises de correlação linear de Pearson, sendo testados até o nível de $5 \%$ de probabilidade.

\section{RESULTADOS E DISCUSSÃO}

Os elevados teores de fósforo orgânico total encontrados nos solos da Ilha de Fernando de Noronha, atingem até 1065 $\mathrm{mg} \mathrm{kg}^{-1}$ (Tabela 3), valores que, mesmo elevados representam,

Tabela 3. Frações de P orgânico (moderadamente lábil-ML e moderadamente resistente-MR), P orgânico total (Pot), frações de $\mathrm{P}$ inorgânico (facilmente solúvel-P- $\mathrm{H}_{2}$ 0, ligado a alumínio-P-Al, ligado a ferro-P-Fe e ligado a cálcio-P-Ca), e teores de $\mathrm{P}$ total dos solos estudados

\begin{tabular}{|c|c|c|c|c|c|c|c|c|c|}
\hline \multirow[t]{2}{*}{ Perfil $^{*}$} & \multirow[t]{2}{*}{ Horiz } & \multirow[t]{2}{*}{ PoML } & PoR & Pot & $\mathrm{P}-\mathrm{H}_{2} \mathrm{O}$ & P-Al & $\mathrm{P}-\mathrm{Fe}$ & $\mathrm{P}-\mathrm{Ca}$ & $\mathrm{Pt}$ \\
\hline & & & \multicolumn{7}{|c|}{$\mathrm{mg} \mathrm{kg}^{-1}$} \\
\hline \multirow{6}{*}{1} & $A p$ & 729 & 0 & 729 & 0 & 23 & 1699 & 113 & 5439 \\
\hline & BA & 0 & 0 & 0 & 0 & 11 & 1802 & 139 & 4983 \\
\hline & Bi1 & 0 & 140 & 140 & 3 & 0 & 1205 & 87 & 4131 \\
\hline & $\mathrm{Bi} 2$ & 0 & 103 & 103 & 0 & 0 & 864 & 51 & 3563 \\
\hline & 2Cvgn & 103 & 0 & 103 & 0 & 0 & 67 & 13 & 515 \\
\hline & A & 0 & 0 & 0 & 1 & 15 & 2526 & 345 & 7352 \\
\hline \multirow{3}{*}{2} & $\mathrm{Bi}$ & 0 & 107 & 107 & 0 & 0 & 1750 & 180 & 5911 \\
\hline & $2 \mathrm{Cnv1}$ & 111 & 39 & 150 & 0 & 0 & 355 & 320 & 1140 \\
\hline & $2 \mathrm{Cnv} 2$ & 0 & 22 & 22 & 0 & 0 & 36 & 3466 & 1532 \\
\hline \multirow{5}{*}{3} & $2 \mathrm{Cr} / \mathrm{R}$ & 0 & 73 & 73 & 0 & 0 & 13 & 2944 & 3168 \\
\hline & A & 100 & 0 & 100 & 2 & 0 & 2760 & 244 & 9247 \\
\hline & Bi1 & 313 & 0 & 313 & 7 & 0 & 3196 & 231 & 9773 \\
\hline & $\mathrm{Bi} 2$ & 347 & 0 & 347 & 2 & 0 & 3294 & 326 & 9372 \\
\hline & C & 188 & 222 & 411 & 1 & 0 & 5420 & 382 & 10750 \\
\hline \multirow{5}{*}{4} & Apn & 701 & 364 & 1065 & 8 & 1 & 1416 & 305 & 3960 \\
\hline & Bin & 225 & 403 & 629 & 12 & 0 & 1047 & 205 & 4290 \\
\hline & Binv & 230 & 0 & 230 & 1 & 3 & 669 & 34 & 2942 \\
\hline & $2 \mathrm{Cnv} 1$ & 91 & 0 & 91 & 1 & 0 & 445 & 38 & 1937 \\
\hline & $2 \mathrm{Cnv} 2$ & 648 & 0 & 648 & 0 & 0 & 82 & 28 & 1140 \\
\hline \multirow{5}{*}{5} & Apn & 0 & 772 & 772 & 15 & 0 & 1639 & 226 & 6105 \\
\hline & Acn & 182 & 356 & 539 & 15 & 0 & 1502 & 96 & 5073 \\
\hline & Cnv & 13 & 0 & 13 & 19 & 0 & 746 & 67 & 3168 \\
\hline & Cnvg1 & 0 & 378 & 378 & 35 & 0 & 468 & 136 & 2646 \\
\hline & Cnvg2 & 0 & 269 & 269 & 10 & 0 & 382 & 185 & 1598 \\
\hline
\end{tabular}

* 1-CAMBISSOLO HÁPLICO Tb Eutrófico vértico gleico Solódico; 2- CAMBISSOLO HÁPLICO Sódico vértico; 3- CAMBISSOLO HÁPLICO Ta Eutrófico típico; 4- CAMBISSOLO HÁPLICO Sódico vértico gleico; 5- VERTISSOLO CROMADO Sódico gleico 
em média, apenas 9,8\% do fósforo total dos solos da ilha chegando, em alguns casos, a valores máximos de 56,8\% do fósforo total. Esta fração orgânica pode ser decisiva na manutenção do $\mathrm{P}$ disponível, visto a maior facilidade de dessorção do $\mathrm{P}$ quando associado a complexos organometálicos, que quando associado a partículas inorgânicas (Zhou et al., 1997).

A fração orgânica lábil (Pol) não apresentou teores detectáveis no fracionamento devido, provavelmente, à condição climática de elevada temperatura e umidade somada à atividade agrícola nesses solos, intensificando os processos de mineralização-imobilização (Chen et al., 2003), os quais contribuem para a instabilidade desta fração nos solos.

Foram encontrados elevados teores de outras frações de fósforo orgânico, com valores variando entre 0 e $729 \mathrm{mg} \mathrm{kg}^{-1}$ para $\mathrm{P}$ orgânico moderadamente lábil, e entre 0 e $772 \mathrm{mg} \mathrm{kg}^{-1}$ para a fração orgânica moderadamente resistente (Tabela 3 ). A presença dessas frações ora na superfície ora em subsuperfície, não pode ser explicada apenas pelas características químicas dos solos, visto que as frações não apresentaram correlação com as características normalmente relacionadas com a acumulação de Po, ou seja, teores de carbono orgânico, fósforo total (Stevenson, 1994) e com os elementos Al e Fe, formando complexos com a matéria orgânica do solo.

A acumulação de fósforo orgânico moderadamente lábil (PoML) nos horizontes subsuperficiais, pode estar relacionada com os elevados teores de argila nesses horizontes, devido à possibilidade de associação das substâncias húmicas aos minerais de argila (Varadachari et al., 1997; Canellas et al., 1999). Os teores constatados de PoML são importantes pelo fato desta fração estar associada aos ácidos fúlvicos (Sequi et., al., 1986), mais facilmente acessados pelas plantas e microrganismos que os ácidos húmicos e, conseqüentemente, disponibilizando $\mathrm{P}$ com maior facilidade.

Assim como foi observado por Cassagne et al. (2000), os teores de fósforo orgânico moderadamente resistente (PoMR) variaram bastante, inclusive entre horizontes do mesmo perfil, resultado que pode estar relacionado com o elevado teor de argila dos horizontes tendo em vista a tendência de acumulação de PoMR nos horizontes em que o teor de argila se eleva; além disso, os teores de PoMR podem estar relacionados com teores de Fe nesses solos, visto que o $\mathrm{NaOH}$, extrator desta fração de $\mathrm{P}$, tem maior eficiência para extrair P ligado a Al e Fe (Novais \& Smyth, 1999). Schlesinger et al. (1998) mostram que esta fração está associada aos ácidos húmicos ou sorvida na superfície dos compostos de Fe e Al.

Nas frações inorgânicas observaram-se teores elevados de $\mathrm{P}-\mathrm{Fe}$, seguidos pelo $\mathrm{P}-\mathrm{Ca}, \mathrm{P}-\mathrm{H}_{2} \mathrm{O}$ e $\mathrm{P}-\mathrm{Al}$ (Tabela 3 ). O fósforo facilmente solúvel $\left(\mathrm{P}-\mathrm{H}_{2} \mathrm{O}\right)$ apresentou, em alguns solos, teores expressivos, variando entre 0 e $35 \mathrm{mg} \mathrm{kg}^{-1}$, conseqüência dos altos teores de $\mathrm{P}$ nesses solos. Os teores $\mathrm{P}-\mathrm{Fe}$, predominante sobre as demais frações, refletem a influência do material de origem dos solos da ilha. Os teores de P-Ca foram inferiores aos de P-Fe, exceto no Cambissolo Háplico sob pastagem nativa (3446 $\mathrm{mg} \mathrm{kg}^{-1}$ ), onde o horizonte $\mathrm{C}$, com elevados teores de $\mathrm{P}-\mathrm{Ca}$, se encontra recoberto pela formação sedimentar dos horizontes superficiais deste perfil.
Mesmo com menor participação nos perfis estudados (9,8\% em média), a correlação obtida entre o P total e o P orgânico total $\left(0,73^{*}\right)$ reflete a importância do compartimento orgânico nesses perfis, visto que o $\mathrm{P}$ orgânico pode estar complexado às substâncias húmicas do solo, com os cátions de $\mathrm{Fe}$ e $\mathrm{Al}$ servindo de ponte (Rheinheimer et al., 1999). As correlações obtidas entre P-Fe e fósforo total $\left(0,95^{*}\right)$ e o fósforo orgânico $\left(0,80^{*}\right)$, além daquelas obtidas com o $\mathrm{P}$ total lábil $\left(0,73^{*}\right), \mathrm{P}$ inorgânico lábil $\left(0,77^{*}\right), \mathrm{P}$ total moderadamente lábil $\left(0,82^{*}\right)$ e $\mathrm{P}$ inorgânico moderadamente lábil $\left(0,83^{*}\right)$, indicam maior participação desta fração nos perfis estudados. Não foram obtidas correlações entre as frações de Pot com P-Fe nem com outra fração inorgânica.

A soma das frações orgânicas e inorgânicas de fósforo estudada, revela-se inferior ao valor de fósforo total, fato que pode ser explicado pela ausência das frações de fósforo ligado a fosfatos de ferro reduzido e fósforo ocluso, previstas no procedimento original de Chang \& Jackson (1957). Referidas frações não foram determinadas por serem não-lábeis e, conseqüentemente, de pouca importância para culturas; no entanto, acumulam altos teores de $\mathrm{P}$, sobretudo em solos mais intemperizados.

Notou-se correlação significativa do P-Ca com o fósforo disponível por Mehlich-1 (Tabela 4), evidenciando a possível superestimação do fósforo disponível pela solubilização da fração ligada a cálcio (Novais \& Smith, 1999), justificando os altos valores de fósforo disponível obtidos com este extrator.

Tabela 4. Correções entre o fósforo disponível por Mehlich 1, Mehlich 3 e Olsen e as frações de P inorgânico (facilmente solúvel $\left(\mathrm{H}_{2} \mathrm{O}\right)$, ligado a ferro, a cálcio e a alumínio) em perfis da ilha grande de Fernando de Noronha

\begin{tabular}{ccccc}
\hline & $\mathrm{H}_{2} \mathrm{O}$ & Ferro & Cálcio & Alumínio \\
\hline Mehlich 1 & $-0,06^{\mathrm{ns}}$ & $0,17^{\mathrm{ns}}$ & $0,86^{* *}$ & $-0,04^{\mathrm{ns}}$ \\
Mehlich 3 & $0,66^{* *}$ & $0,47^{*}$ & $-0,36^{\mathrm{ns}}$ & $-0,10^{\mathrm{ns}}$ \\
Olsen & $0,07^{\mathrm{ns}}$ & $0,77^{* *}$ & $-0,40^{*}$ & $-0,29^{\mathrm{ns}}$ \\
\hline ns - Não significativo; & siginifitativo a $5 \%{ }^{* *}$ siginifitativo a $1 \%$ &
\end{tabular}

Quando se utiliza o Mehlich-3 como extrator, observam-se valores menores dos encontrados com o Mehlich 1 (Tabela 5), com correlações significativas com as frações consideradas lábeis, ou seja, fósforo facilmente solúvel, $\mathrm{P}$ inorgânico lábil $\left(0,50^{*}\right)$, P inorgânico moderadamente lábil $\left(0,67^{*}\right)$ (estes últimos obtidos no fracionamento do Po), além do P-Fe $\left(0,47^{*}\right)$, indicando a capacidade do extrator em estimar melhor o fósforo disponível.

O fósforo disponível pelo extrator Olsen apresenta correlação com o $\mathrm{P}-\mathrm{Fe}\left(0,77^{*}\right)$, principal componente do $\mathrm{P}$ total e de maior solubilidade que as demais frações, e fósforo total do solo $\left(0,81^{*}\right)$, indicando que este também é melhor estimador do fósforo dísponível que o Mehlich 1, visto que não foram obtidas correlações entre este último e as frações de fósforo, exceto P-Ca (Tabela 4). Além dos valores distintos, não se obtiveram correlações entre os valores de fósforo disponível pelos extratores Mehlich 1, Mehlich 3 e Olsen. 
Tabela 5. Fósforo disponível por Mehlich 1, Mehlich 3 e Olsen em perfis de Fernando de Noronha

\begin{tabular}{|c|c|c|c|c|}
\hline Perfil* & Horiz. & Mehlich 1 & $\frac{\text { Mehlich } 3}{\left(\mathrm{mg} \mathrm{kg}^{-1}\right)}$ & Olsen \\
\hline \multirow{5}{*}{1} & Ap & 349 & 87 & 282 \\
\hline & $\mathrm{BA}$ & 270 & 102 & 277 \\
\hline & Bi1 & 338 & 104 & 277 \\
\hline & $\mathrm{Bi} 2$ & 145 & 49 & 158 \\
\hline & 2 Cvgn & 29 & 2 & 26 \\
\hline \multirow{5}{*}{2} & $\mathrm{~A}$ & 270 & 114 & 247 \\
\hline & $\mathrm{Bi}$ & 259 & 56 & 265 \\
\hline & $2 \mathrm{Cnv} 1$ & 31 & 5 & 50 \\
\hline & $2 \mathrm{Cnv} 2$ & 1002 & 2 & 12 \\
\hline & $2 \mathrm{Cr} / \mathrm{R}$ & 1053 & 12 & 11 \\
\hline \multirow{4}{*}{3} & A & 338 & 143 & 208 \\
\hline & Bil & 497 & 115 & 324 \\
\hline & $\mathrm{Bi} 2$ & 418 & 101 & 353 \\
\hline & $\mathrm{C}$ & 611 & 291 & 274 \\
\hline \multirow{5}{*}{4} & Apn & 346 & 248 & 254 \\
\hline & Bin & 401 & 201 & 233 \\
\hline & Binv & 74 & 80 & 95 \\
\hline & $2 \mathrm{Cnv} 1$ & 54 & 73 & 58 \\
\hline & $2 \mathrm{Cnv} 2$ & 54 & 83 & 56 \\
\hline \multirow{5}{*}{5} & Apn & 243 & 262 & 252 \\
\hline & Acn & 188 & 256 & 263 \\
\hline & $\mathrm{Cnv}$ & 228 & 172 & 167 \\
\hline & Cnvg1 & 314 & 250 & 102 \\
\hline & Cnvg2 & 2 & 110 & 55 \\
\hline
\end{tabular}

* 1- CAMBISSOLO HÁPLICO Tb Eutrófico vértico gleico Solódico; 2- CAMBISSOLO HÁPLICO Sódico vértico; 3- CAMBISSOLO HÁPLICO Ta Eutrófico típico; 4- CAMBISSOLO HÁPLICO Sódico vértico gleico; 5- VERTISSOLO CROMADO Sódico gleico

\section{CONCLUSÕES}

1. Os altos teores de fósforo observados nos solos de Fernando de Noronha são decorrentes principalmente do material de origem.

2. O fósforo orgânico foi menos importante que as formas inorgânicas na contribuição para o fósforo total desses solos.

3. P-Fe e P-Ca são as frações predominantes nos solos estudados.

4. Os extratores Mehlich 3 e Olsen são mais indicados que o Mehlich 1, para estimar o P disponível nos solos estudados.

\section{AGRADECIMENTOS}

À Coordenação de Aperfeiçoamento de Pessoal de Nível Superior-CAPES, e ao Programa de Pós-Graduação em Ciência do Solo da UFRPE.

\section{LITERATURA CITADA}

Bowman, R.A. A sequential extraction procedure with concentrated sulfuric acid and dilute base for soil organic phosphorus. Soil Science Sociey of America Journal, Madison, v.53, n.2, p.362-366, 1989.
Bowman, R.A.; Cole, C.V. An exploratory method for fractionationof organic phosphorus from grassland soils. Soil Science, Baltimore, v.125, n.2, p.95-101, 1978.

Braga, J.M.; Defelipo, B.V. Determinação espectrofotométrica de fósforo em extratos de solos e plantas. Revista Ceres, Viçosa, v.113, n.1, p.73-85, 1974.

Canellas, L.P.; Santos, G.A.; Amaral Sobrinho, N.M.B. Reações da matéria orgânica In: Santos, G.A.; Camargo, F.A.O. (ed). Fundamentos da matéria orgânica do solo: ecossistemas tropicais e subtropicais. Porto Alegre: Gênesis, 1999. p.6989.

Cassagne, N; Remaury, M; Gaulquelin, T; Fabre, A. Forms and profile distribution of soil phosphorus in alpine Iceptisols and Spodosols (Pyrennes, France). Geoderma, Amsterdam, v.95, n.2, p.161-172, 2000.

Chang, S.C.; Jackson, M.L. Fractionation of soil phosphorus. Soil Science, Baltimore, v.84, p.133-144, 1957.

Chen, C.R.; Condron, L.M.; Davis, M.R.; Sherlock, R.R. Seazonal changes in soil phosphorus and associated microbial properties under adjacent grassland and forest in New Zealand. Forest Ecology and Management, Amsterdam, v.177, n.3, p. 539-557, 2003

Cross, A.F.; Schlesinger, W.H. A literature review and evaluation of the Hedley fractionation: Applications to the biogeochemical cycle of soil phosphorus in natural ecossistems. Geoderma, Amsterdam, v.64, n.3, p.197-214, 1995.

Duda, G.P. Conteúdo de fósforo microbiano, orgânico e biodisponível em diferentes classes de solo. Seropédica: UFRRJ, 2000. 158p. Tese Doutorado

EMBRAPA - Empresa Brasileira de Pesquisa Agropecuária. Manual de métodos de análises de solo. Brasília: 1997, 212p.

EMBRAPA - Empresa Brasileira de Pesquisa Agropecuária. Sistema brasileiro de classificação de solo. Brasília: 1999, 219p.

Guerra, J.G.M. Produção sazonal de Brachiaria decumbens Stapf. conteúdo de fósforo orgânico e microbiano em solos de baixa produtividade natural. Seropédica: UFRRJ, 1993. 234p. Tese Doutorado

Guerra, J.G.M. Conteúdo de fósforo orgânico em amostras de solo. Pesquisa Agropecuária Brasileira, Brasília, v.31, n.2, p.291-299, 1996.

Lemos, R.C.; Santos, R.D. Manual de descrição e coleta de solo no campo. 3.ed. Campinas: Sociedade Brasileira de Ciência do Solo, 1996. 83p.

Mehlich, A. Mehlich 3 soil test extractant: A modification of Mehlich 2 extractant. Communications of Soil Science and Plant Analisys, New York, v.15, n.12, p.1049-1416,1984.

Nascimento, C.W.A. Fertilidade dos solos da Ilha de Fernando de Noronha. In: Reunião Brasileira de Fertilidade do Solo e Nutrição de Plantas - FERTBIO, 25, 2002, Rio de Janeiro. Anais... Rio de Janeiro: UFRRJ, 2002. CD-ROM

Novais, R.F. Phosphourus supplying capacity of previously heavily fertilizad soils. Raleigh: North Caroline State University, 1977. 151p. PhD Thesis

Novais, R.F.; Smith, T.J. Fósforo em solo e planta em condições tropicais. Viçosa: DPS/UFV, 1999. 399p.

O‘Halloran, I.P. Total and organic phosphorus. In: Carter, M.R. (ed) Soil sampling and methods of analysis. Boca Roton: Lewis Publisching, 1993.p.213-229. 
Olsen, S.R.; Cole, C.V.; Watanabe, F.S.; Dean, L.A. Estimation of available phosphorus in soils by extraction with sodium bicarbonate. Washington: USDA/USGPO, 1954. 32p.

Rheinheimer, D.; Cassol, P.C.; Kaminski.; Anghinoni, I. Fósforo orgânico do solo. In: Santos, G.A.; Camargo, F.A.O. (ed.). Fundamentos da matéria orgânica do solo: ecossistemas tropicais e subtropicais. Porto Alegre: Gênesis, 1999. p.6989.

Schlesinger, W.H.; Bruilnzeel, L.A.; Bush, M.B.; Klein, E.M.; Mace, K.A.; Raikes, J. A.; Whittaker, R.J. The biogeochemistry of phosphorus after the first century of soil development on Rakata Island, Krakatau, Indonesia. Biogeochemistry, Dordrecht, n.40, n.1, p.37-55. 1998.

Segui, P.; Nobili, M.; Leita, L.; Cercignani, G. A new index of humification. Agrochimica, Pisa, v.30, p.175-179, 1986.

Silva, F.C.; Raij, B. van. Disponibilidade de fósforo avaliada por diferentes extratores. Pesquisa Agropecuária Brasileira, Brasília, v.34, n.12, p.2277-2285, 1999.
Smeck, N.E. Phosphorus dynamics in soils and landscapes. Geoderma, Amsterdam, v.36, n.3, p.185-199, 1985.

Stevenson, F.J. Húmus chemistry. New York: John Willey, 1994. 496p.

Tan, K.H. Soli sampling, preparation, and analysis. New York: Marcel Dekker, 1996. 408p.

Tiessen, J.; Stewart, J.W.B.; Cole, C.V. Pathways of phosphorus transformations in soils of differing pedogenesis. Soil Science Society of America Jounal, Madison, v.48, n.4, p. 853-858, 1984.

Varadachari, C.; Chattopadhyay, T.; Ghosh, K. Complexation of humic substances with oxides of iron and aluminum. Soil Science, Baltimore, v.162, n.1, p.28-34, 1997.

Walker, T.W.; Syers, J.K. The fate of phosphorus during pedogenesis. Geoderma, Amsterdam, v.15, p.1-19, 1976.

Zhou, X.; Rhue, R.D.; Harris, W.G. Phosphorus sorption characteristics of $\mathrm{Bh}$ and $\mathrm{Bt}$ horizons from sandy coastal palin soils. Soil Science Society of America Journal, Madison, v.61, n.5, p.1364-1369, 1997. 